\title{
Identification and Wide-area Visualization of the Centers of Oscillation for a Large-scale Power System
}

\author{
Leonardo E. Bernal, Fengkai Hu, Kai Sun \\ University of Tennessee \\ Knoxville, TN, USA \\ leo.bernal@gatech.edu \\ fengkaihu@utk.edu \\ kaisun@utk.edu
}

\author{
Evangelos Farantatos \\ Electric Power Research Institute \\ Palo Alto, CA, USA \\ efarantatos@epri.com
}

\begin{abstract}
A system wide disturbance event usually triggers interarea oscillations at the system. Understanding the nature of those oscillations is important for taking necessary early mitigation actions. This work presents a novel scheme for analyzing interarea oscillations by identification of the centers of oscillation (CoO) using two methods based on wide-area measurement data: one method directly calculate CoOs for transmission lines with synchrophasors on both ends and the other estimate CoOs based on contour visualization about frequency deviations extrapolated for the entire map. CoOs serve as pivots between oscillating parts under disturbances and hence indicate the grid interfaces involved in oscillation, so this scheme can provide the operator recommendations on where mitigation actions may be taken to prevent instability. The proposed scheme is tested using simulated synchrophasor data on a WECC 179-bus test system.
\end{abstract}

Index Terms-- center of oscillation; inter-area oscillation; out-ofstep; phasor measurement unit; synchrophasor; wide-area visualization

\section{INTRODUCTION}

A SERIOUS problem that has plagued the electrical transmission networks of the world since the beginning of mass power generation is large scale blackouts. An ideal power system maintains the frequency at exactly $60 \mathrm{~Hz}$. However a disturbance will cause the system frequency to oscillate. The system is expected to experience more frequency oscillations in the future when intermittent energy resources, such as solar and wind, are integrated in the system. Multiple, sequential faults can lead to a power outage. However, these do not happen instantaneously, and certain electrical system conditions must gradually worsen with time for such an event to occur. The reliability of the grid decreases when angular oscillations increase [1][2]. Such angular instability in the system can result in out of step (OoS) generators and system separation. An example is the August 10, 1996 Western Interconnection cascading event.

This material is based upon work supported partially by the US Department of Energy, the National Science Foundation, and the CURENT Engineering Center research and development funding
In order to reduce the occurrence of future cascading events, situational awareness is critical for the system operators. With this information, the best operation needed can be taken to ensure or recover system stability. Wide area situational awareness has been enabled by synchrophasors, e.g. Phasor Measurement Units (PMUs).

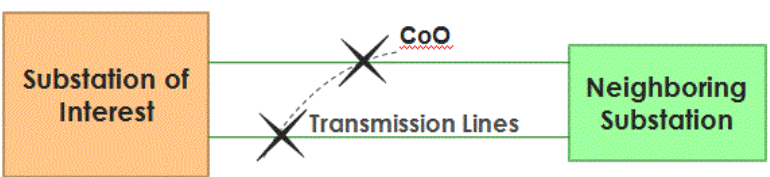

Fig. 1. Local center of oscillation located at points in the transmission lines some distance between neighboring substations.

Real-time analysis of system inter-area oscillations can be achieved by generation coherency analysis or identification of the oscillation mode shape using PMU data, as proposed in [2]. This paper investigates a new approach, i.e. the identification of the center of oscillation ( $\mathrm{CoO}$ ) for the dominant inter-area oscillation mode as illustrated by Fig. 1 [3]. CoOs can be defined as the physical locations where the rate of change of frequency is constant. They serve as "pivots" of frequency oscillations between interconnected regions. It must be noted that multiple CoOs may exist in a system, which correspond to multiple oscillation modes, either local or inter-area.

Based on the theoretical foundation of CoOs presented in [3] and [4], this paper investigates the visualization of $\mathrm{CoOs}$ for a large-scale power system. Those CoOs appearing with slowfrequency oscillations caused by disturbances actually indicate the weakest grid interfaces vulnerable to angular instability or severe generation out of step. Accordingly, this paper proposes a new scheme for analyzing system interarea oscillations by the identification and visualization of the CoOs. Dynamically, weak transmission lines can be found in the system in real time indicated by the located CoOs. Visualization of those $\mathrm{CoOs}$ in real time, together with the information on the inter-area 
oscillation path proposed in [5], will help improve operators' situational awareness especially during cascading events.

Two methods for $\mathrm{CoO}$ identification is presented and tested in this paper: one method uses synchrophasor measurements from both ends of individual transmission lines based on the definition of a $\mathrm{CoO}$ and the other method utilizes wide-area visualization and contour map of synchrophasor data.

\section{Proposed Visualization TeChNiQUe}

A major component of the proposed scheme is monitoring and visualization of wide-area system frequencies or frequency deviations, measured by a PMU-based wide-area measurement system or the frequency monitoring network (FNET) which serves the North American power grid with real-time frequency measurements, visualization and event alerts [6]. Some works on wide-are power system frequency visualization are such as [7] and [8].

A MATLAB program was developed to monitor the widearea frequency oscillations and tested using a simplified WECC (Western Interconnection) 179-bus power system network as shown in Fig. 2.

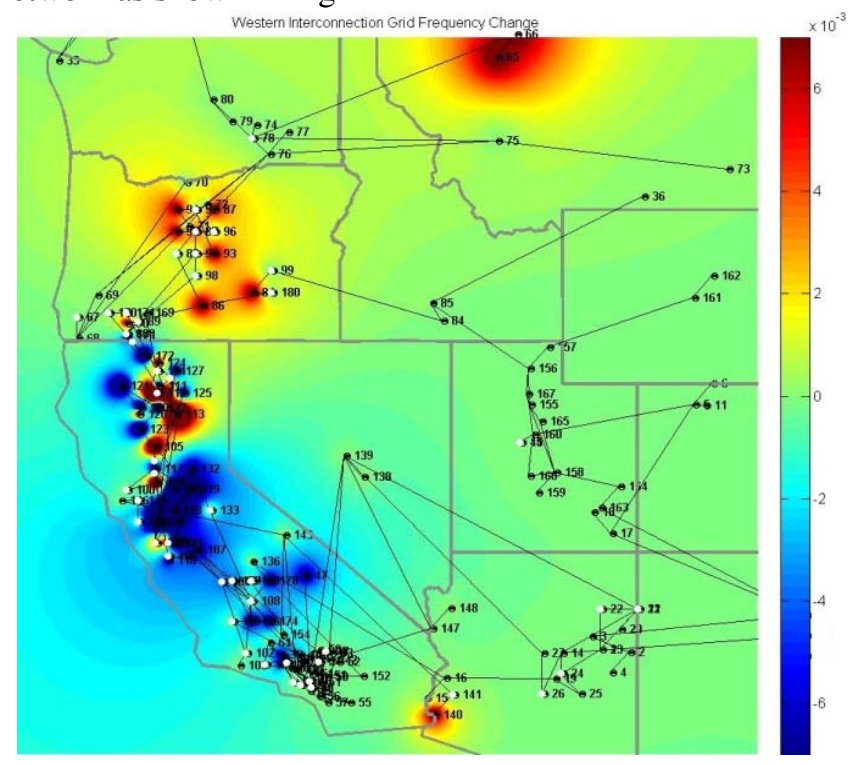

Fig. 2. Wide-area visualization of frequency deviations in the WECC 179-bus system with $\mathrm{CoOs}$ identified on transmission lines

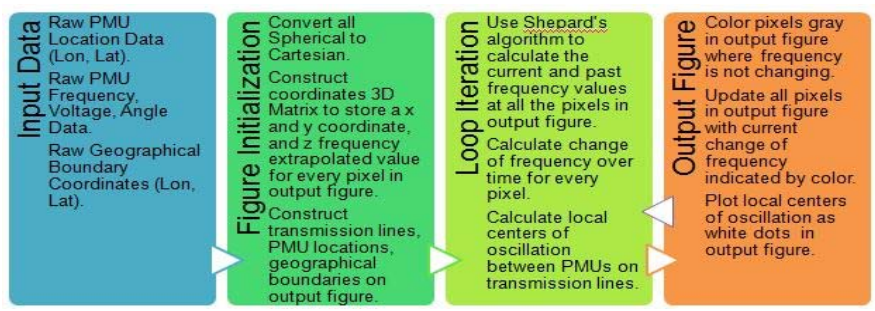

Fig. 3. Universal code structure of the visualization program

The program structure, shown in Fig. 3, receives simulated PMU data from each of the 179 buses in the network as an input and breaks down the data to time, voltage, angle and frequency components. A separate header file contains the PMU latitude and longitude locations that are converted to Cartesian by the Miller cylindrical projection method [9] due to the matrix nature of the calculations. The accuracy in distances of the transmission lines between buses will vary depending on the map projection method and are assumed to perfectly straight and level between buses, important factors in determining the position of the $\mathrm{CoO}$ and accounting for error. The WECC 179-bus network was constructed as a column matrix of two rows that contains all the possible bus pairs in the network that are directly connected by a transmission line. This is used to draw the transmission lines and for the purpose of calculating a $\mathrm{CoO}$ between two buses.

At every pixel there is an extrapolated value on frequency, voltage or angle depending on the user's choice. A two dimensional matrix was created to hold all the values. The index of each value represents the location of the pixel value. The minimum and maximum coordinates of the PMU locations were determined to set the matrix limits. A mesh grid function was then used to create three $\mathrm{x}, \mathrm{y}$, and $\mathrm{z}$ matrices with resolution of 864 by 623 pixels as shown in Fig. 4 .

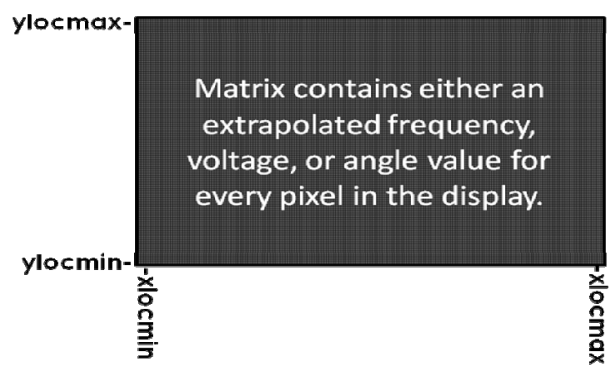

Fig. 4. Universal display matrix has an extrapolated value for the frequency, voltage, or angle at every pixel, or row/column index.

Shepard's algorithm was implemented to extrapolate frequency points outside the PMU locations on the map [10][11]. For each pixel point $(x, y)$ in display without an actual PMU, the extrapolated frequency at time $t$, denoted by $f_{(x, y), t}$, has a quadratic inverse relationship with the distance to each PMU, whose location is denoted by $\left(x_{i}, y_{i}\right)$, and a linear proportional relationship with the frequency measurement $f_{(x, y), t}$ as follows

$$
f_{(x, y), t}=\sum_{i=1}^{n} \frac{K_{\left(x_{i}, y_{i}\right), t} \times f_{\left(x_{i}, y_{i}\right), t}}{\left(x-x_{i}\right)^{2}+\left(y-y_{i}\right)^{2}} /\left[\sum_{i=1}^{n} \frac{K_{\left(x_{i}, y_{i}\right), t}}{\left(x-x_{i}\right)^{2}+\left(y-y_{i}\right)^{2}}\right]
$$

where $n$ is the total number of PMUs, and $K_{(x x i y i), t}$ is a weight factor for each PMU. The default value of the weight factor is 1 for all PMUs. If a PMU is found with low-quality (e.g. missing or noisy) data, its weight factor will be decreased or simply equal 0 to exclude it from the $\mathrm{CoO}$ visualization to avoid generating wrong information. As shown in Fig. 3, after the initial conditions of the plot are set, the program will iterate through PMU data. For every time step, e.g. 1-2 cycles depending on the PMU sampling rate, it will calculate the current frequency pixel values based on measured frequencies. After the frequencies are extrapolated for all pixels, a medianvalue filter is applied to the results to remove noise without significantly reducing the sharpness of frequency changes.

\section{COO CALCULATION}

The next step is to determine where a $\mathrm{CoO}$ may be located 
in the system. Two methods, called the local $\mathrm{CoO}$ method and the pixel method, are utilized in the program. For each transmission line with PMUs on both ends, the local $\mathrm{CoO}$ method calculates the $\mathrm{CoO}$ directly on that line based on the algorithm introduced below; the pixel method estimates CoOs as the locations with a constant rate of change of frequency based on the frequencies extrapolated for the entire map at the pixel level. Later, the test on the 179-bus system shows that the results of two methods basically match with each other especially for a dominant inter-area oscillation mode. The pixel method will be illustrated by coloring the pixels in respect to the rate of change of frequency with green color for constant change.

Since the power system at the continental scale is excessively nonlinear and convoluted system, the time frame must be small in order to assume a linear relationship as shown in Fig. 5. $f_{1}$ and $f_{2}$ are the frequencies from two buses directly connected by a transmission line. $\mathrm{f}_{\mathrm{CoO}}$ is the linear function of the $\mathrm{CoO}$ [3]. A PMU records frequency measurements at $0.0333 \mathrm{~s}$ intervals for $30 \mathrm{~Hz}$ sampling rate. Here, 5 consecutive data points, i.e. over 0.167 seconds, are used to maintain an approximate linear relationship between frequency and distance.

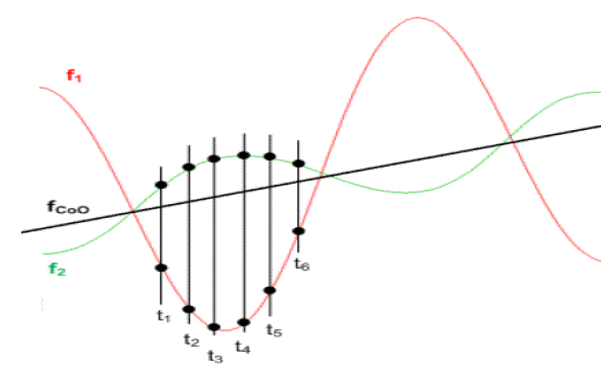

Fig. 5. Center of oscillation linearity principle

Therefore to find the location of the local $\mathrm{CoO}$ point along the transmission line, several assumptions are made.

- The CoO function is approximately linear within the five data points.

- The frequency will be linear along the transmission line as it varies with time and travel from one PMU to the other.

- Transmission lines are perfectly straight from one PMU to the other.

- $\mathrm{CoO}$ exists within the line.

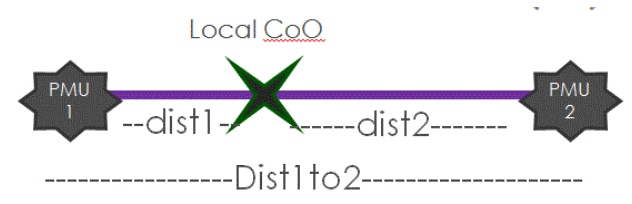

Fig. 6. Center of Oscillation Calculation Method

With these, an equation can be derived to calculate the CoO. As shown in Fig. 2, after the initial conditions of the plot are set, the program will iterate through the simulated PMU data. For every $0.0333 \mathrm{~s}$, it will calculate the current $\mathrm{CoO}$ that may be in any of the transmission lines.

$$
\Delta f_{1, \text { avg }}=\frac{\sum_{j=1}^{m}\left(f_{\left(x_{i}, y_{i}\right), t-j+1}-f_{\left(x_{i}, y_{i}\right), t-j}\right)}{m}
$$

For one iteration of the code, an attempt is made to find a $\mathrm{CoO}$ along the line of all the pairs of PMUs that are directly connected by a transmission line. The derivative is taken of the frequency of the PMU pair. $m$ (i.e. 5) data points are considered again to match the pixel rate of frequency method.

$$
\operatorname{dist}_{1}=\frac{\left|\Delta f_{1, \text { avg }}\right| \times \text { dist }_{1-2}}{\left|\Delta f_{1, \text { avg }}\right|+\left|\Delta f_{2, a v g}\right|}
$$

Then the distance of $\mathrm{CoO}$ can be found from one of the PMU pair using the linear relationship equation and is stored in variable dist ${ }_{1}$. The frequency of PMU 1 corresponds to $d f_{1, \text { avg }}$ and similarly PMU 2 frequency with $d f_{2, \text { avg. The distance }}$ between the PMU pair can be easily found to determine the coordinates of the local CoO. If $d f_{1, a v g}$ and $d f_{2, \text { avg }}$ have opposite signs, the $\mathrm{CoO}$ is plotted in the display since this indicates an oscillation between the PMU buses. For the final result, both the local $\mathrm{CoO}$ method and pixel method are shown in the display along with the contour map.

\section{CASE STUDY}

The simplified WECC system is used to examine the proposed method. The system contains 29 generators, 179 buses, and 263 branches. Assume that at every bus, there is a PMU. To simulate a cascading event, six three phase faults happen respectively at $0 \mathrm{~s}$ (line 83-172), $40 \mathrm{~s}$ (line 114-124), $80 \mathrm{~s}$ (line 115-130), $120 \mathrm{~s}$ (line 114-170), $160 \mathrm{~s}$ (line 83-94), and at $200 \mathrm{~s}$ (line 83-98). Faults are controlled after six cycles of their fault time by tripping the fault line. However, each contingency weakens the system's ability to return to equilibrium which accumulates in widespread instability after the 6th fault [12]. Fig. 7 gives some insight to the increasing vulnerability of area-wide system due to a chain of contingencies. Each figure shows the system frequency in a $3 \mathrm{D}$ space. The vertical axis shows the frequency and ranges from $59.9 \mathrm{~Hz}$ to $60.1 \mathrm{~Hz}$, while the horizontal axes shows the Cartesian coordinates of the plane. The elevation of a point in the plane indicates its respective frequency. The 3-D visualization shows how every point pulls on the overall frequency in either direction thus affecting area-wide stability. Fig. 7(a) displays a stable system after two faults. By the aftermath of the fourth fault, the system has developed a dangerous cycle of oscillation shown in Fig. 7(b)-(d) demonstrate the culmination of these circumstances that ends in a major area blackout.

In order to effectively distinguish regions that are in oscillation, pixels are colored by the rate of change of frequency over time at the pixel locations as demonstrated in Fig. 2. For these simulations, five points starting from the current time and separated by a $0.033 \mathrm{~s}$ time step were used to calculate the derivation. Green regions indicate a constant rate of change of frequency, which are approximate $\mathrm{CoO}$ locations. Red regions and blue regions indicate increasing and decreasing frequency, respectively, and are the regions oscillating against each other. The figure color bar ranges from $-0.7 \mathrm{~Hz} \cdot \mathrm{s}^{-1}$ to $0.7 \mathrm{~Hz} \cdot \mathrm{s}^{-1}$. White dots on the black transmission lines represent the CoOs seen on those lines based on the local 
CoO method. Fig. 2 demonstrates the CoOs identified by two methods for a normal operating condition. Note that the scheme proposed in this paper does not differentiate inter-area and local models, so the CoOs visualized are about all visible oscillation modes. In Fig. 2, lots of white dots are dispersed close to generator buses, which include some local plant mode $\mathrm{CoOs}$, and the green region (having a constant rate of change of frequency) spreads out in the entire system, indicating the oscillation under that condition has not developed to a systemwide problem. However, once one inter-area mode grows to become dominant and lead to a system-wide oscillation problem, the white dots (CoOs by the local $\mathrm{CoO}$ method) will aggregate around the oscillation boundary of that mode and the green region (CoOs by the pixel method) will also match that boundary well, as illustrated by Fig. 9 .

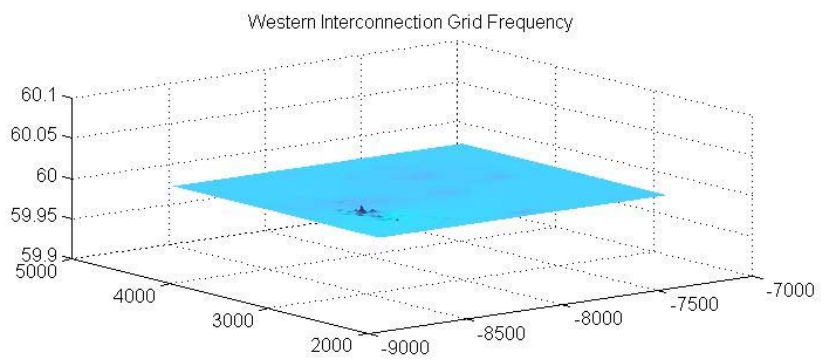

(a) At $\mathrm{t}=67.12 \mathrm{~s}$.

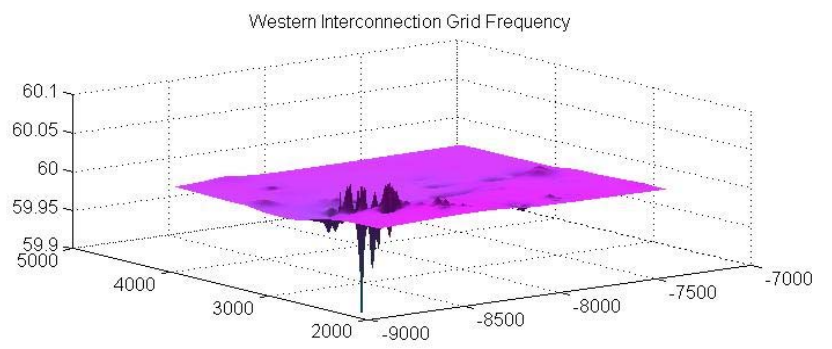

(b) At $\mathrm{t}=126.32 \mathrm{~s}$

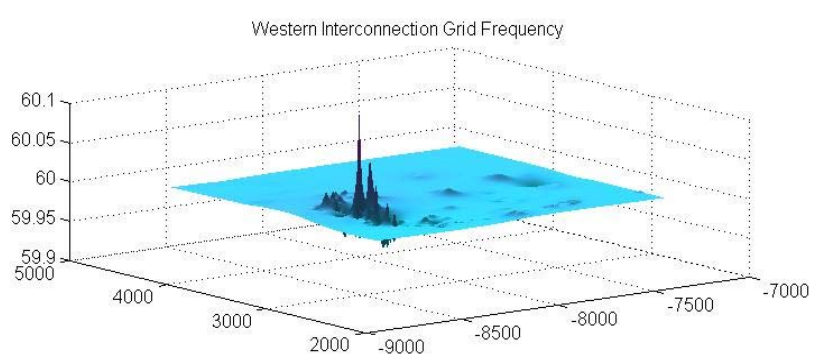

(c) At $\mathrm{t}=195.33 \mathrm{~s}$

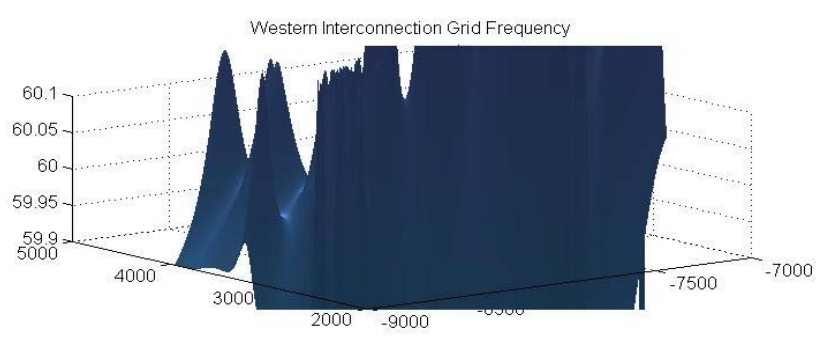

(d) At t $=207.24 \mathrm{~s}$

Fig. 7. Rate of change of frequency of the 179-bus system in 3D display

The 188 s to 208 s period was exclusively examined for system oscillation behaviors. Before the sixth fault at 200s, the system was observed to have a consistent oscillation pattern as shown by the four snapshots in Fig. 8, where the white dot on each transmission line is the local CoO located by the using the PMU data from two ends of the line, and the color contours are about frequency deviations at each point of the map based on interpolation or extrapolation. The green color on the contour map indicates the inter-area $\mathrm{CoO}$ boundary based on the frequency deviations. From this information, it is clear that the northern and southern regions always have opposite rates of frequency change. Therefore, a $\mathrm{CoO}$ boundary splitting up the two major northern and southern regions can be obtained from the real-time PMU data. A clearer picture of this boundary is shown in Fig. 9, when the system is going to collapse into two electrical islands. The $\mathrm{CoO}$ boundary seen by the green color of the visualization is consistent well with the local CoOs indicated by the white dots. Those local CoOs or other lines with green color indicate where control may be added to mitigate the stability problem. One strategy could be to perform controlled intentional separation of the grid into electrical islands with mitigation actions to stabilize each formed island according to a previous study [12]. By examining what transmission lines intersect with the $\mathrm{CoO}$ boundary, the locations of controlled separation may be determined.

In practice, a power utility may not place PMUs at all buses. Thus, the local $\mathrm{CoO}$ method only works for the lines with PMUs on both ends. However, the pixel method will still work to provide the locations of $\mathrm{CoOs}$ with decreased accuracy depending on the density of PMUs. In Fig. 10, two visualizations respectively with PMUs at $50 \%$ of buses and PMUs only at 29 generator buses are shown for the same condition as Fig. 8(a). The first visualization matches very well with Fig. 8(a). The second one shows a slightly different contour map but can still give approximate locations of the $\mathrm{CoO}$ boundary in terms of the transmission lines involved

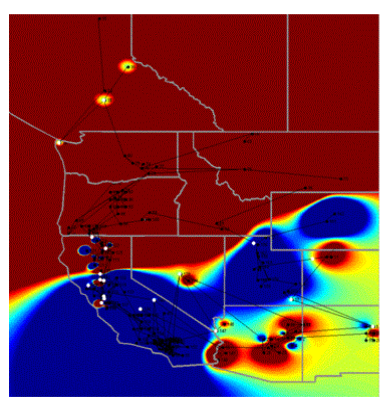

(a)

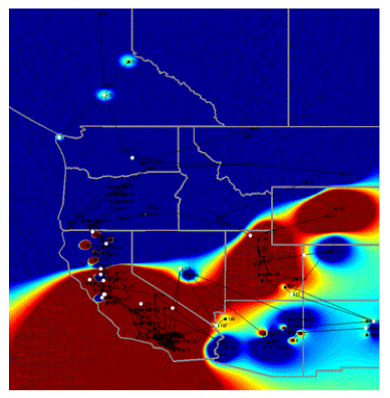

(c)

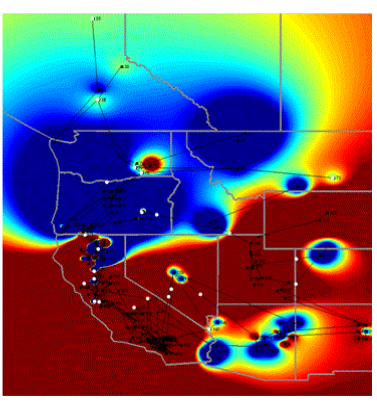

(b)

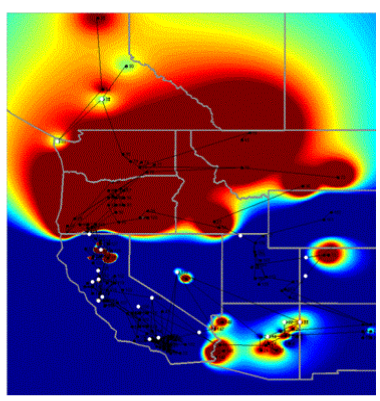

(d)
Fig. 8. WECC 179-bus oscillation shown in order from stages (a) to (d) 


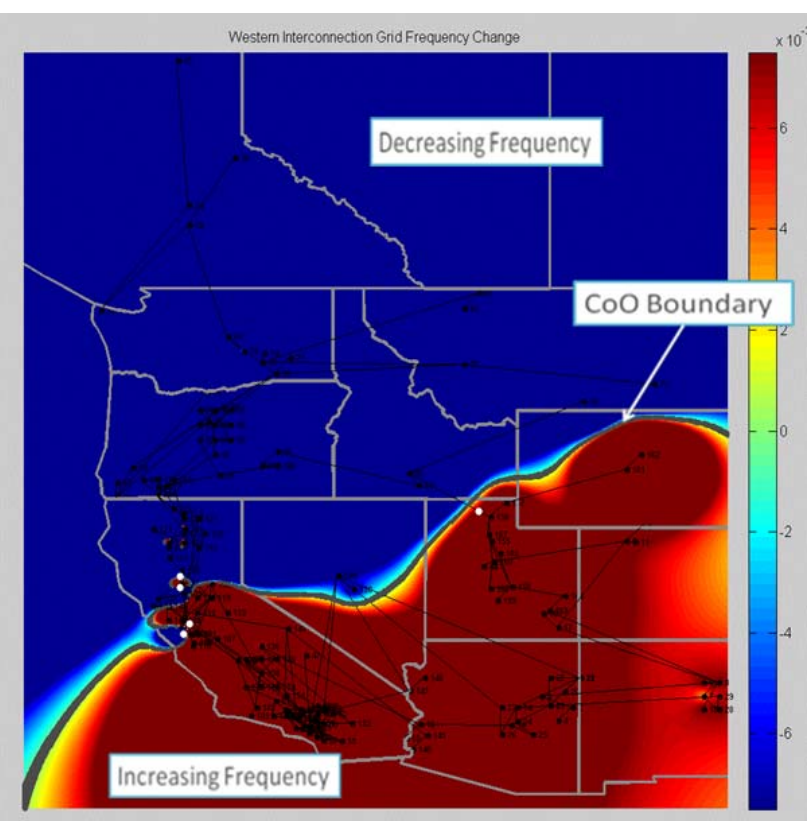

Fig. 9. CoO boundary between two islanded systems in oscillation

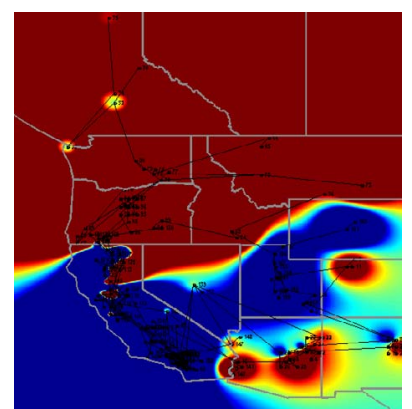

(a) PMUs at $50 \%$ buses

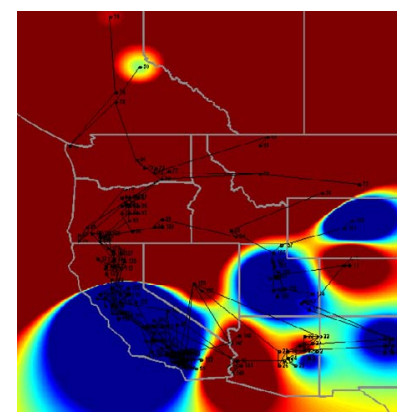

(b) PMUs at 29 generators
Fig. 10. Center of Oscillation boundary identified by the pixel method

V.

\section{CONCLUSIONS}

A scheme and related software program were designed to visualize real time synchrophasor measurements in the form of contour displays and identify the $\mathrm{CoO}$ locations that serve as pivots of the frequency oscillations between interconnected regions. The following can be concluded: 1) results can be used to characterize and predict potential angular instability; 2) Using the $\mathrm{CoO}$ location of $\mathrm{CoOs}$, the critical grid interfaces or weakest transmission lines can be identified in electrical grid for immediate remedial actions; 3) It may help operators identify and analyze inter-area oscillations and improve situational awareness.

\section{REFERENCES}

[1] J. Hauer, et al, "Integrated Dynamic Information for the Western Power System: WAMS Analysis in 2005", in book: Power System Stability and Control: The Electric Power Engineering Handbook, CRC, 2007

[2] K. Sun, X. Luo, J.M. Wong, "Early Warning of Wide-Area Angular Stability Problems Using Synchrophasors", IEEE PES General Meeting, San Diego, CA, 23-26 July 2012

[3] E. Farantatos, "A Predictive Out-of-Step Protection Scheme Based on PMU Enabled Distributed Dynamic State Estimation," Ph.D. dissertation, Sch. ECE, Georgia Tech., Atlanta., GA, 2013.

[4] E. Farantatos, et al, "Real-Time Power System Dynamic Equivalencing to Preserve System Center of Oscillations via PMU-Based Dynamic
State Estimator", IEEE PES General Meeting, Vancouver, BC, Canada, July $21-25,2013$

[5] Y. Chompoobutrgool, et al, Identification of Power System Dominant Inter-Area Oscillation Paths, IEEE Trans. Power System, vol. 28, No. 3, pp. 2789-2807, Aug 2013.

[6] Y. Zhang, et al, "Wide-area frequency monitoring network (FNET) architecture and applications," IEEE Trans. Smart Grid, Vol. 1, No. 2, pp. 159-167, Sept. 2010.

[7] G. Zhang, P. Hirsch and S. Lee, "Wide Area Power system visualization Using Smart Client Technology", IEEE PES General Meeting, Tampa, Florida, 2007.

[8] G. Zhang, K. Sun, et al, "Application of Synchrophasor Measurements for Improving Operator Situational Awareness", IEEE PES General Meeting, Detroit, MI, 24-29 July 2011

[9] Flattening the Earth: Two Thousand Years of Map Projections, John P. Snyder, 1993, pp. 179, 183, ISBN 0-226-76747-7.

[10] D. Shepard, "A two-dimensional interpolation function for irregularlyspaced data," in Proc. of the 23rd ACM National Conference, New York, NY, 1969, pp. 517-523

[11] D. O. Savageau, et al, "Adaptive influence distance algorithm for contouring bus-based power system data," Annual Hawaii International Conference on System Sciences, Kona, Hawaii, January 3-6, 2007.

[12] K. Sun, K. Hur, P. Zhang, "A New Unified Scheme for Controlled Power System Separation Using Synchronized Phasor Measurements," IEEE Trans. Power Systems, vol.26, no.3, pp.1544,1554, Aug. 2011

\section{BIOGRAPHIES}

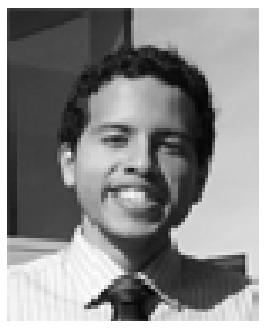

Leonardo Bernal was born in Bogota, Colombia where he spent his early childhood before moving to Weston, Florida. $\mathrm{He}$ has performed undergraduate research in the field of beta-voltaics at Georgia Tech Lorraine in Metz, France. He did a Research Experience for Undergraduates at University of Tennessee Knoxville, TN in the summer of 2013. He is currently working towards a B.S. degree in electrical engineering at Georgia Institute of Technology.

Fengkai Hu received the B.S. and M.S. degrees in automation from University of Electronic Science and Technology of China, Chengdu, China, in 2009 and 2012, respectively. He is currently working toward the Ph.D. degree in electrical engineering in University of Tennessee, Knoxville, TN, USA. His research interests include wide area measurement system visualization, voltage and frequency stability, smart grid communication and wide area system protection and control.

Kai Sun (M'06-SM'13) received the B.S. degree in automation and the Ph.D. degree in control science and engineering from Tsinghua University, Beijing, China, in 1999 and 2004, respectively. He was a postdoctoral research associate at Arizona State University, Tempe, from 2005 to 2007, and was a project manager in grid operations and planning areas at EPRI, Palo Alto, CA from 2007 to 2012. He is currently an assistant professor at the Department of EECS, University of Tennessee in Knoxville.

Evangelos Farantatos (St. M '06, M '13) was born in Athens, Greece in 1983. He received the Diploma in Electrical and Computer Engineering from the National Technical University of Athens, Greece, in 2006 and the M.S. in E.C.E. and Ph.D. degrees from the Georgia Institute of Technology in 2009 and 2012 respectively. He is currently a Sr. Project Engineer/Scientist at EPRI. In summer 2009, he was an intern in Midwest ISO. His research interests include power systems state estimation, protection, stability, operations, control, synchrophasor applications, renewables integration and smart grid technologies. 